\title{
A Brief Introduction to Nomography: Graphical Representation of Mathematical Relationships
}

\author{
Leslie Glasser* \\ Nanochemistry Research Institute, Department of Chemistry \\ Curtin University, GPO Box U1987, Perth, WA 6845, Australia \\ and \\ Ron Doerfler \\ Advisory Systems Engineer, Naperville, Illinois, 60565 USA \\ 6 Figures 1 Table 3 Online Resource Files $\sim 3000$ words
}

\section{Keywords:}

Graduate Education/Research, Continuing Education, Interdisciplinary / Multidisciplinary, Mathematics / Symbolic Mathematics, Nomograph, Nomogram, Alignment Chart, Graphic Aids

*Corresponding author

L. Glasser: $\quad$ Telephone: $+6189848-3334$

E-mail:1.glasser@curtin.edu.au

ORCiD : 0000-0002-8883-0564 
This is an Accepted Manuscript of an article published by Taylor \& Francis in International Journal of Mathematical Education in Science and Technology on 26/09/2018 available online at:

https://www.tandfonline.com/doi/full/10.1080/0020739X.2018.1527406

\section{Abstract}

Nomographs (or nomograms, or alignment charts) are graphical representations of mathematical relationships (extending to empirical relationships of data) which are used by simply applying a straightedge across the plot through points on scales representing independent variables, which then crosses the corresponding datum point for the dependent variable; the choice among independent and dependent variable is arbitrary so that each variable may be determined in terms of the others. Examples of nomographs in common current use compute the lift available for a hot-air balloon, the boiling points of solvents under reduced pressure in the chemistry laboratory, and the relative forces in a centrifuge in a biochemical laboratory. Sundials represent another ancient yet widely-familiar example. The origins and geometry of the nomograph are seldom currently understood or taught.

With the advent and ready accessibility of the computer, printed mathematical tables, slide rules and nomographs became generally redundant. However, there remains even today a place for nomographs since they provide insight into mathematical relationships, are useful for rapid and repeated application (as illustrated by the examples listed above), even in the absence of calculational facilities, and can reliably be used in the field even by nonspecialists. Many nomographs for various purposes may be found online.

This paper describes the origins and development of nomographs, and illustrates their use with some relevant examples. A supplementary interactive Excel file demonstrates their application for some simple mathematical operations. 
This is an Accepted Manuscript of an article published by Taylor \& Francis in International Journal of Mathematical Education in Science and Technology on 26/09/2018 available online at:

https://www.tandfonline.com/doi/full/10.1080/0020739X.2018.1527406

\section{Introduction}

In the years BC - "Before Computers", that is - the principal modes of calculation were by use of printed mathematical tables[1,2] such as for logarithms and trigonometric functions, or by use of slide-rules, or by graphical means in the form of pre-prepared nomographs.[3, 4, 5] With the advent of computers, printed tables often became redundant - although the NIST Digital Library of Mathematical Functions[6] and its printed companion still remains as a fundamental source of reference, together with such esoteric resources as the "Online Encyclopedia of Integer Sequences"[7] - while slide-rules have generally disappeared. Nomographs (from the Greek "nomo" = "law"), or nomograms, or alignment charts although the latter terms have become widely subverted to other applications - appeared to become obsolescent in the 1950s, but have remained available, generally in specialist fields, and may even be undergoing a partial renaissance for their speed of use, ready accessibility and reliability, even if their construction is seldom currently discussed or understood. It is the purpose of this paper to introduce the reader to nomography, to the construction of nomographs, and to some of their applications.

Nomography[8] was developed in 1884 by Philbert Maurice d'Ocagne (in dealing with cutand-fill problems in the construction of French railways) as a graphic solution for multivariable functions, being an extension of two-variable Cartesian graphs, with the orthogonal axes transformed into separated parallel axes, as seen in Fig. 1. Based on the principles of projective geometry:

"d'Ocagne showed that a point on a graph with Cartesian coordinates transformed into a line on [a nomogram], that a line transformed into a point, and, finally, that a family of lines or a surface transformed into a single line... The nomogram has several great advantages. First, it allows for great economy of expression: the diagram is much less 
This is an Accepted Manuscript of an article published by Taylor \& Francis in International Journal of Mathematical Education in Science and Technology on 26/09/2018 available online at:

https://www.tandfonline.com/doi/full/10.1080/0020739X.2018.1527406

cluttered than a graph with Cartesian coordinates. Second, the same nomogram can express all the parameters of a formula and can handle many more variables. And third, one reads off the values on a nomogram by ... laying a straightedge across the scales and noting where it intercepts a third scale, thereby greatly increasing the speed and accuracy of reading the graph. There is no need to follow a line from the abscissa to the curve to the ordinate, as is the case with Cartesian coordinates.'[9]

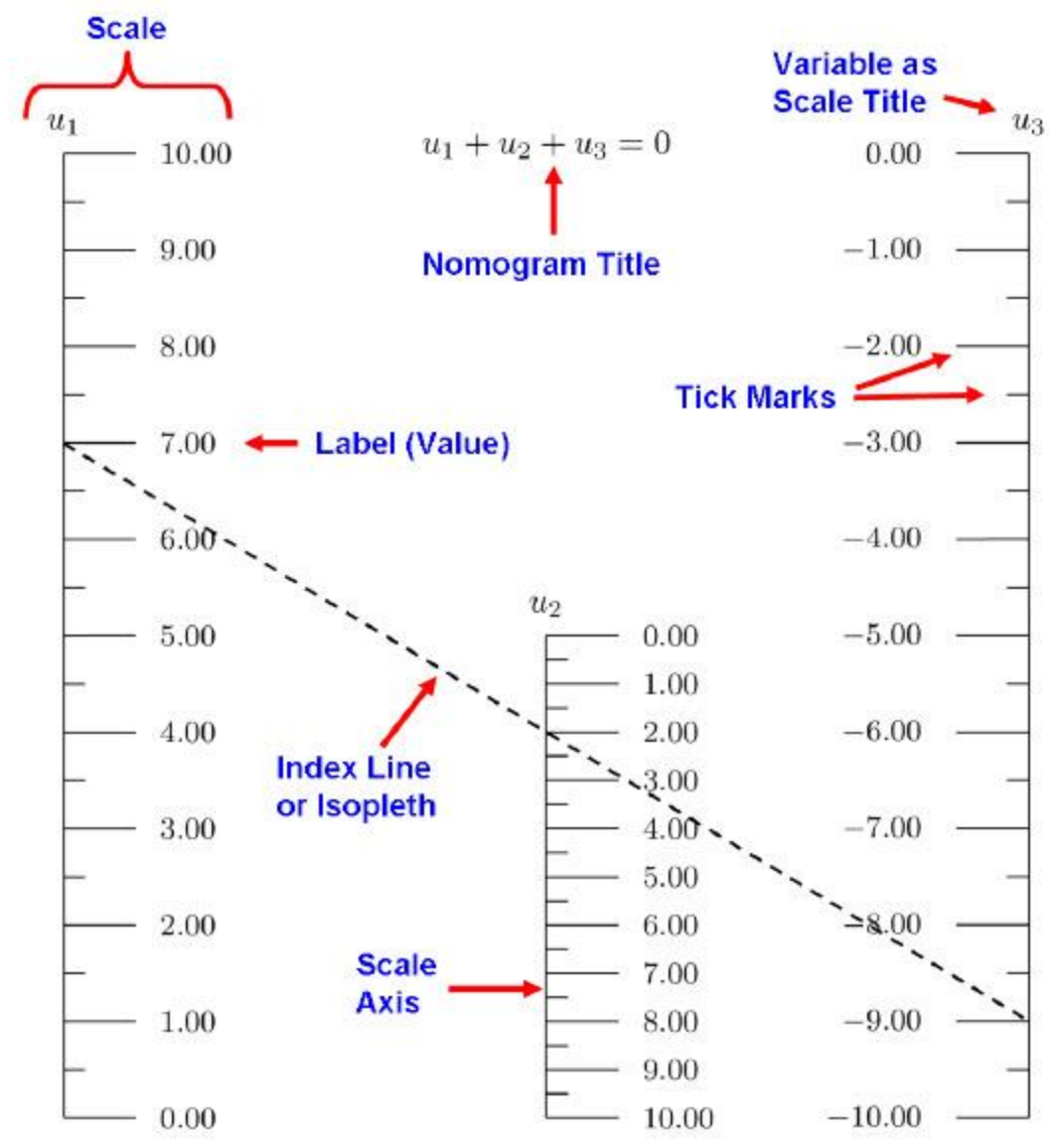

Figure 1: A linear parallel 3-axes nomograph for addition $\left(u_{1}+u_{2}+u_{3}=0\right)$, prepared using the nomograph-generating program PyNomo[10] based on Python program text. The broken straight line joining the data points $\left(u_{1}=7, u_{3}=-9\right.$, with resultant negated sum $\left.u_{2}=2\right)$ is an instance of an isopleth.[11] Reprinted with permission from Doerfler, R. http://www.myreckonings.com/pynomo/CreatingNomogramsWithPynomo.pdf. 
This is an Accepted Manuscript of an article published by Taylor \& Francis in International Journal of Mathematical Education in Science and Technology on 26/09/2018 available online at:

https://www.tandfonline.com/doi/full/10.1080/0020739X.2018.1527406

While the simple form of a nomograph for addition, such as Fig. 1, seems trivial, it becomes more useful when it is recalled than many multiplicative functions can be converted into additions by taking logarithms of the functions. An example of a practical application (Fig. 2) is in calculating the relative force in a rotating centrifuge[12] where

$$
\mathrm{RCF} / g=1.118 \cdot 10^{-5} r N^{2}(\mathrm{RCF} \equiv \text { Relative Centrifugal Force })
$$

with radius, $r$, in centimeters, and rotational velocity, $N$, in revolutions per minute, so that

$$
\log (\mathrm{RCF})=-5+\log (1.118)+\log (r)+2 \log (N)
$$

A much elaborated nomograph, which also takes into account the time of centrifugation for consideration of the processes of sedimentation, has been published by Rasmussen.[13] 
This is an Accepted Manuscript of an article published by Taylor \& Francis in International Journal of Mathematical Education in Science and Technology on 26/09/2018 available online at:

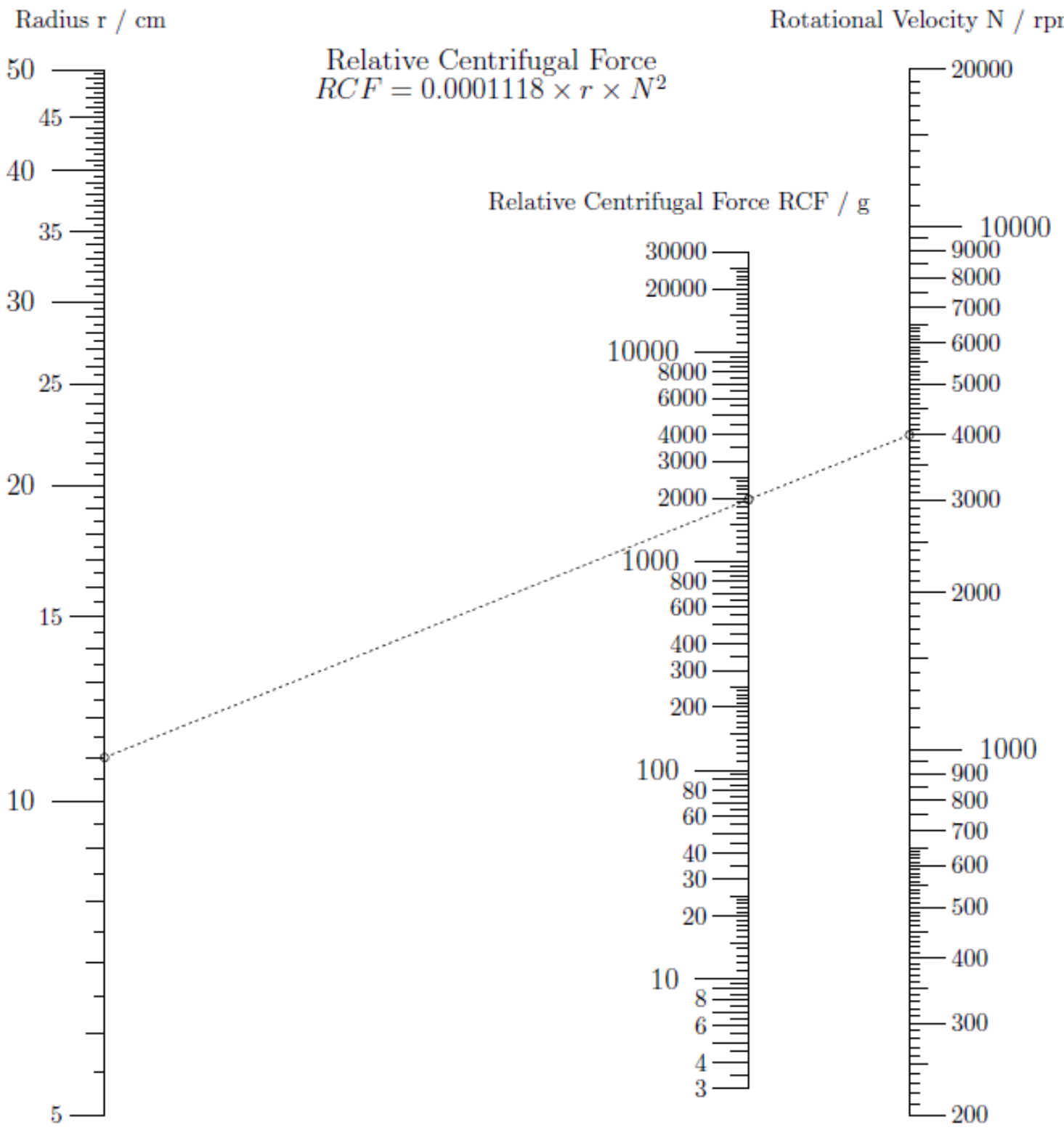

Figure 2: A three parallel-scale nomograph for determining the relative force in a rotating centrifuge with RCF/ $g=1.118 \cdot 10^{-5} r N^{2}(\mathrm{RCF} \equiv$ Relative Centrifugal Force), created using PyNomo.[10, 11] 
This is an Accepted Manuscript of an article published by Taylor \& Francis in International Journal of Mathematical Education in Science and Technology on 26/09/2018 available online at:

https://www.tandfonline.com/doi/full/10.1080/0020739X.2018.1527406

\section{Hot-Air Balloon Load Chart}

Hot-air balloonists must take appropriate care that their balloons can successfully lift their payload. The necessary basic information is provided by a loading chart nomograph (also known as a network chart)[14] based on the ideal gas formula, a balloon envelope temperature of $100^{\circ} \mathrm{C}$, and the atmospheric pressure at various altitudes (Fig. 3). An online calculator version is also available, but does not currently respond![15] This emphasises an important issue with regard to online facilities, which may not always be available[16] for a variety of reasons or which become obsolete through software changes, unlike a printed nomograph. 


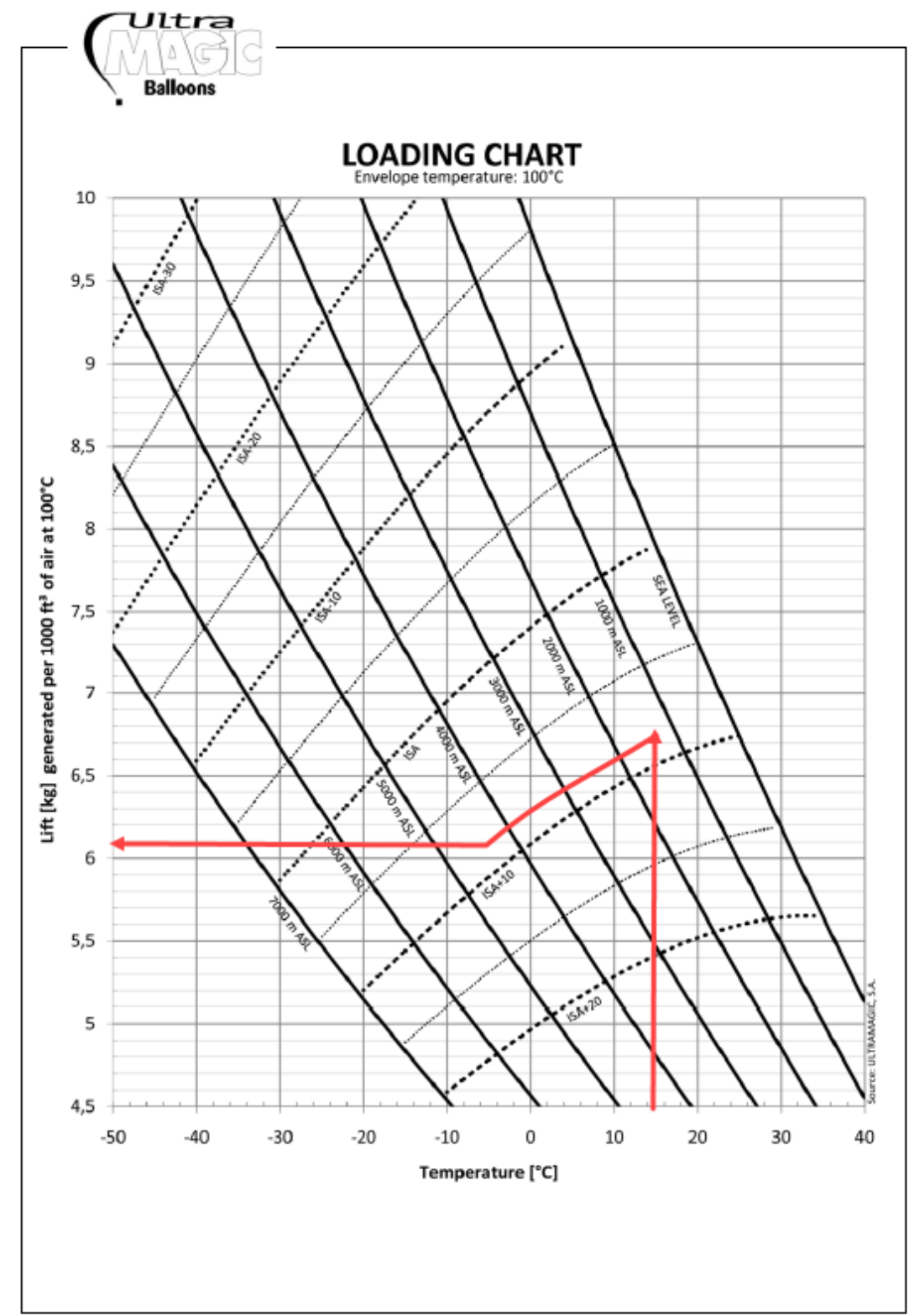

Figure 3: A loading chart for hot-air balloonists, showing the lift generated at ground level for a balloon envelope temperature of $100^{\circ} \mathrm{C}$ at various altitudes from sea level to $7000 \mathrm{~m}$ ASL (Above Sea Level), for various values of the International Standard Atmosphere ( ISA) of $15{ }^{\circ} \mathrm{C}$ and 1013.2 millibars (mb, or $\mathrm{hPa}$ ). In use, select the ambient temperature (abscissa), follow vertically to the current altitude, then follow parallel to the ISA temperature line to the desired maximum altitude and, finally, look horizontally to the available lift (lines added by authors). Published with the permission of Ultramagic Balloons, http://ultramagic.com/openfiles/Manuals04/LoadChart.Pdf. 
This is an Accepted Manuscript of an article published by Taylor \& Francis in International Journal of Mathematical Education in Science and Technology on 26/09/2018 available online at:

https://www.tandfonline.com/doi/full/10.1080/0020739X.2018.1527406

\section{An Updated Vapour Pressure Nomograph}

A vapour pressure nomograph is in common use in chemical laboratories, based on two parallel temperature axes and a third curved pressure axis.[17, 18] The basic nomograph is calculated using the thermodynamic Clausius-Clapeyron equation although this is seldom mentioned in the resources. Of course this equation has limitations, including assumptions of temperature-independent enthalpy of vaporisation, negligible liquid volume, ideal behaviour of the vapour and inapplicability to associating materials, and so the nomograph is further modified for greater accuracy[19] (see Fig. 4). Assessment is rapidly made without the necessity of any computing device. 


\section{Pressure-Temperature Nomograph}

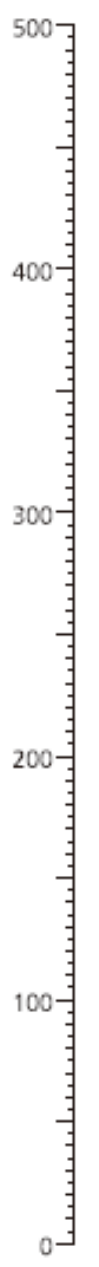

[C]

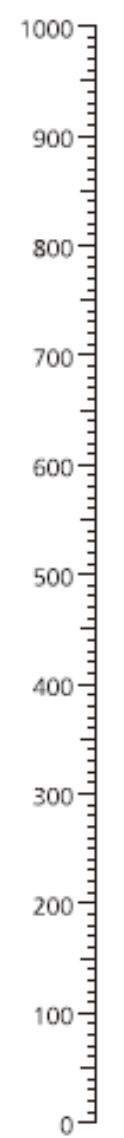

$760 \mathrm{mmHg}$

$(101,32 \mathrm{kPa})$

B

A

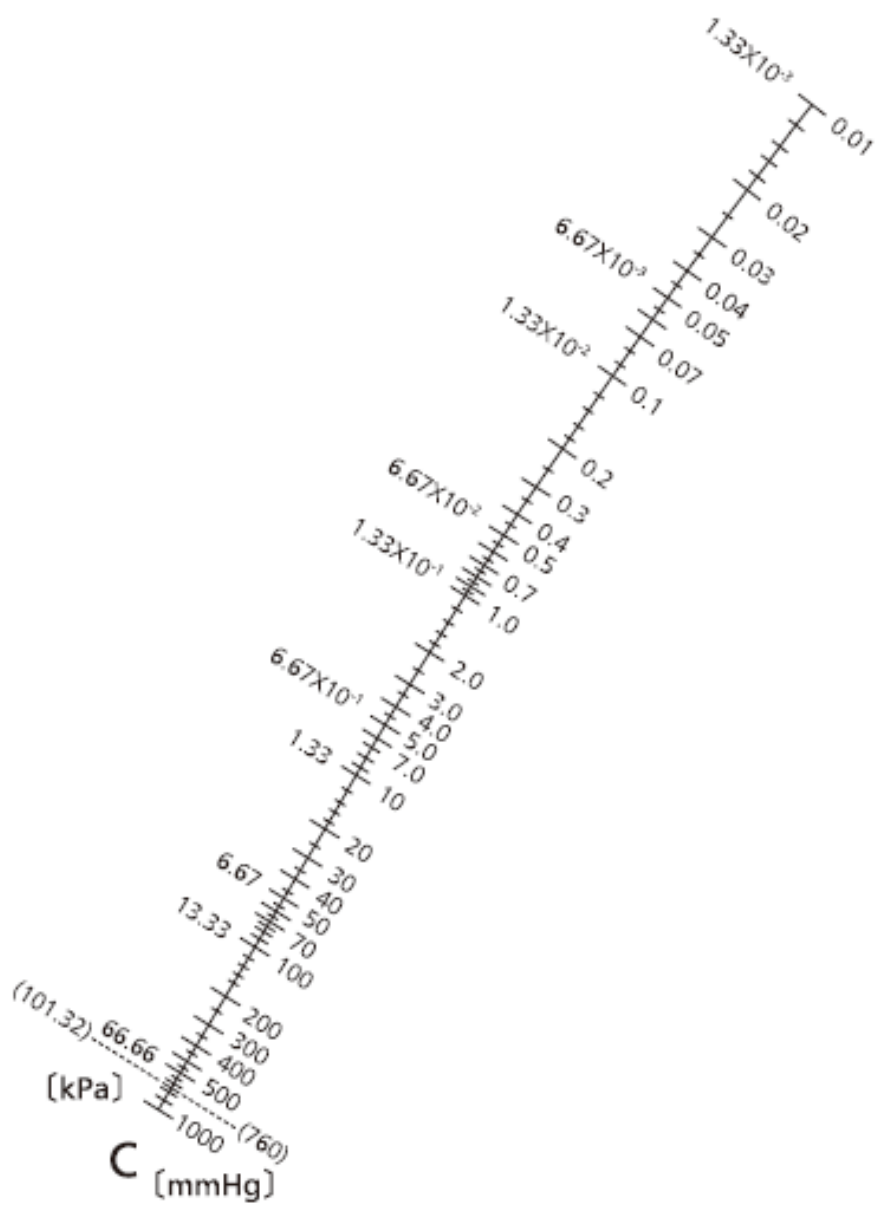

- How to calculate the bp under atmospheric pressure from bp under reduced pressure

(1) Connect a degree on the line $\mathrm{C}$ and its corresponding bp on the line $\mathrm{A}$ under reduced pressure using a straight line.

(2) An intersection found by step (1) on the line B serves as an approximate bp in atmospheric pressure.

* This nomograph applies to nonassociated solvent.

Since the bp obtained from this nomograph is an approximate value, it is not an exact bp.

Reference : Science of Petroleum, Vol.II. p.1281 (1938).

Figure 4: Pressure-Temperature nomograph[18] adapted from

http://www.tcichemicals.com/pdf/pressure-temperature-nomograph.pdf. Published with the kind permission of TCI (Tokyo Chemical Industry) www.TCIchemicals.com 
This is an Accepted Manuscript of an article published by Taylor \& Francis in International Journal of Mathematical Education in Science and Technology on 26/09/2018 available online at:

https://www.tandfonline.com/doi/full/10.1080/0020739X.2018.1527406

\section{Geometry and Types of Nomographs[3, 20, 21]}

Figure 5 shows the most basic parallel-scale nomograph, for addition.

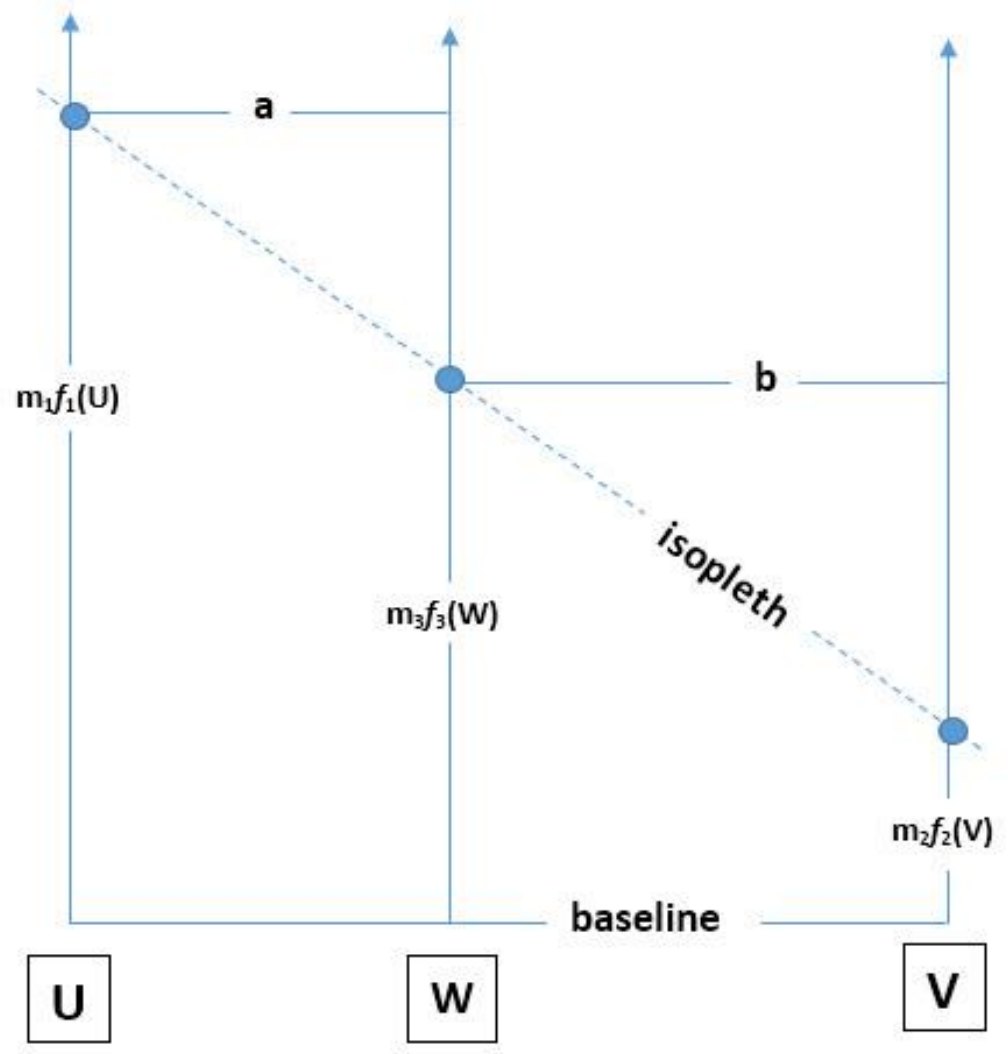

Figure 5: The basic parallel-scale nomograph[20] for the calculation of a sum: $m_{3} f_{3}(W)=$ $m_{1} f_{1}(U)+m_{2} f_{2}(V)$, with scale factors $m_{1}, m_{2}$, and $m_{3}$.

By similar triangles, we have the relations among the scale factors which satisfy the sum $\mathrm{m}_{3} f_{3}(\mathrm{~W})=\mathrm{m}_{1} f_{1}(\mathrm{U})+\mathrm{m}_{2} f_{2}(\mathrm{~V}):$

$$
\frac{m_{1} f_{1}(U)-m_{3} f_{3}(W)}{a}=\frac{m_{3} f_{3}(W)-m_{2} f_{2}(V)}{b}
$$

$$
\text { Thus } \frac{m_{1}}{m_{2}}=\frac{a}{b} \text { and } m_{3}=\frac{m_{1} m_{2}}{m_{1}+m_{2}}
$$


This is an Accepted Manuscript of an article published by Taylor \& Francis in International Journal of Mathematical Education in Science and Technology on 26/09/2018 available online at:

https://www.tandfonline.com/doi/full/10.1080/0020739X.2018.1527406

Using this information, a parallel-scale nomograph may be set up. If the range of $\mathrm{W}$ is very small, its scale may be expanded by moving it outside the outer scales.

In general, nomographs may be prepared using determinants, provided that the determinant can be set up in the generic Massau form:[22, 23]

$$
\left|\begin{array}{lll}
f_{1}\left(x_{1}\right) & g_{1}\left(y_{1}\right) & 1 \\
f_{2}\left(x_{2}\right) & g_{2}\left(y_{2}\right) & 1 \\
f_{3}\left(x_{3}\right) & g_{3}\left(y_{3}\right) & 1
\end{array}\right|=0
$$

This determinant yields the area of a triangle in the $x y$-plane. When the area is zero, the triangle corresponds to the straight-line isopleth with coordinates $\left(x_{1}, y_{1}\right),\left(x_{2}, y_{2}\right)$ and $\left(x_{3}, y_{3}\right)$, independent of the actual values of $x$ and $y$, and so can be arbitrarily placed across the nomograph to yield the desired result.

These principles may be used to establish nomographs of various kinds. The generic PyNomo software[10] is programmed to be able to prepare 10 different kinds of nomograph, as listed in Table 1.

Table 1: Nomograph types available for construction by PyNomo:[10] equation form, nomograph form, rough sketch. ${ }^{\text {a }}$

\begin{tabular}{|c|c|c|c|}
\hline Type & Form of Equation & $\begin{array}{c}\text { Form of } \\
\text { Nomograph }\end{array}$ & Sketch \\
\hline 1 & $f_{1}\left(u_{1}\right)+f_{2}\left(u_{2}\right)+f_{3}\left(u_{3}\right)=0$ & $\begin{array}{c}\text { Three Parallel } \\
\text { Scales }\end{array}$ & Z or N Chart \\
\hline 2 & $f_{1}\left(u_{1}\right)=f_{2}\left(u_{2}\right) \times f_{3}\left(u_{3}\right)$ & Compound \\
\hline 3 & $f_{1}\left(u_{1}\right)+f_{2}\left(u_{1}\right)=f_{2}\left(u_{2}\right) / f_{3}\left(u_{3}\right)+\ldots+f_{n}\left(u_{n}\right)=0$ &
\end{tabular}


This is an Accepted Manuscript of an article published by Taylor \& Francis in International Journal of Mathematical Education in Science and Technology on 26/09/2018 available online at:

https://www.tandfonline.com/doi/full/10.1080/0020739X.2018.1527406

\begin{tabular}{|c|c|c|c|}
\hline & $\begin{array}{c}f_{1}\left(u_{1}\right)+f_{2}\left(u_{2}\right)+f_{3}\left(u_{3}\right)=0 \text { and } f_{3}\left(u_{3}\right)+ \\
f_{4}\left(u_{4}\right)+f_{5}\left(u_{5}\right)=0\end{array}$ & $\begin{array}{l}\text { Parallel Scales } \\
3 \text { Parallel } \\
\text { Scales } \\
\text { Compounded }\end{array}$ & \\
\hline 4 & $f_{1}\left(u_{1}\right) / f_{2}\left(u_{2}\right)=f_{3}\left(u_{3}\right) / f_{4}\left(u_{4}\right)$ & Proportion & \\
\hline 5 & $f_{1}(v)=f_{2}(x, u)$ & Contour & \\
\hline 6 & $u=u$ & Ladder & \\
\hline 7 & $1 / f_{3}\left(u_{3}\right)=1 / f_{1}\left(u_{1}\right)+1 / f_{2}\left(u_{2}\right)$ & $\begin{array}{l}\text { Reciprocal / } \\
\text { Angle }\end{array}$ & \\
\hline 8 & $y=f(u)$ & Single Scale & \\
\hline 9 & $\left|\begin{array}{lll}f_{1}\left(u_{1}\left[, v_{1}\right]\right) & g_{1}\left(u_{1}\left[, v_{1}\right]\right) & h_{1}\left(u_{1}\left[, v_{1}\right]\right) \\
f_{2}\left(u_{2}\left[, v_{2}\right]\right) & g_{2}\left(u_{2}\left[, v_{2}\right]\right) & h_{2}\left(u_{2}\left[, v_{2}\right]\right) \\
f_{3}\left(u_{3}\left[, v_{3}\right]\right) & g_{3}\left(u_{3}\left[, v_{3}\right]\right) & h_{3}\left(u_{3}\left[, v_{3}\right]\right)\end{array}\right|$ & $\begin{array}{l}\text { General } \\
\text { Nomogram } \\
\text { based on } \\
\text { Determinant }\end{array}$ & \\
\hline 10 & $f_{1}\left(u_{1}\right)+f_{2}\left(u_{2}\right) \times f_{3}\left(u_{3}\right)+f_{4}\left(u_{3}\right)=0$ & $\begin{array}{l}\text { One Curved } \\
\text { Line }\end{array}$ & \\
\hline
\end{tabular}

a Adapted from "Creating Nomograms with the PyNomo Software" by Ron Doerfler, 2009. http://www.myreckonings.com/pynomo/CreatingNomogramsWithPynomo.pdf

The standard nomograph relates three variables and has three corresponding scales. However, these are by no means the only nomograph types which can be created. Allowance for more than three variables may be made by linking nomographs together in a series. In addition to the above mathematically-based variations, many of the published early nomographs were developed by empirical means, as follows. Set up an arbitrary pair of parallel scales and triangulate points on the third scale; that is, draw an isopleth across the two scales which points towards the third value (which has been calculated by some relevant equation, or from 
This is an Accepted Manuscript of an article published by Taylor \& Francis in International Journal of Mathematical Education in Science and Technology on 26/09/2018 available online at:

https://www.tandfonline.com/doi/full/10.1080/0020739X.2018.1527406

an experimental datum), then draw a second isopleth pointing to the same third value (for

example: $6=2+4$ and $6=3+3$ may be the values on the two original scales for an

addition). Where the two isopleths cross is the coordinate for the desired third point; this

procedure is repeated to generate a range of third-value coordinates through which a smooth

curve may be drawn to complete the nomograph. Since the crossing may occur at slightly

different points for various choices of isopleth pairs, one may choose a centre of a set of

crossing points and adjust the position of one of the original scales. This is done iteratively to establish an optimum nomograph.

\section{Comparison of Surface Plots, Contour Plots and Nomographs}

Graphic representations of data provide insight into the relative influences of the variables in a functional relationship which are obscured in tables of data. Surface and contour plots provide such information for three-variable systems in a readily assimilable form (supplementary Fig. S1 and S2 are surface and contour plots of the Body Mass Index, BMI).

However, attempting to obtain numeric values from such plots is awkward, and nomographs[24] provide a convenient alternative view (Fig. 6). 


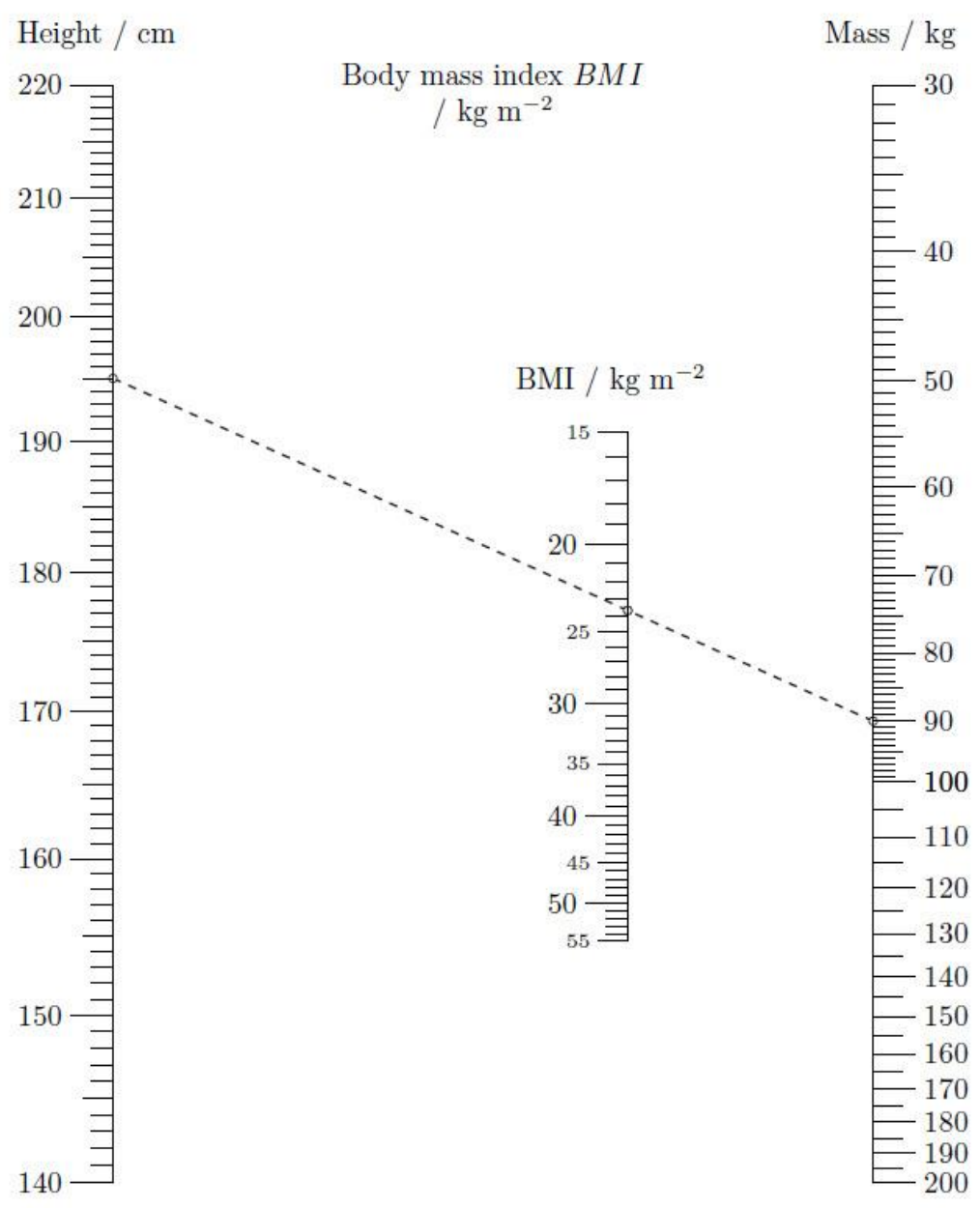

Figure 6: A Body Mass Index Nomograph, BMI / $\mathrm{kg} \mathrm{m}^{-2}=10000\left(\mathrm{M} / \mathrm{H}^{2}\right)$, created using PyNomo. $[10,11]$

Examination of the nomograph, by considering isopleths radiating from, say, a given weight shows how height enters into the relation, and vice versa, while also providing numeric data. While it might be considered that the BMI is a relatively trivial nomographic application, a recent extended BMI nomograph provides corrections to the possibility of drug overdose for obese children.[25] Indeed, the problems of incorrect dosage in hospitals are matters of great concern, $[26,27]$ with nomographs providing a reliable means for dosage calculation in a clinical situation. 
This is an Accepted Manuscript of an article published by Taylor \& Francis in International Journal of Mathematical Education in Science and Technology on 26/09/2018 available online at:

https://www.tandfonline.com/doi/full/10.1080/0020739X.2018.1527406

\section{Other Application Areas}

One significant nomographic application is the elaborate (and, indeed, beautiful) Smith chart, $[28,29,30]$ which continues to be used by electrical engineers for calculations relating to transmission lines and impedance matching. An ancient application of nomography (even if not generally recognised as such) is on sundials,[31] where the upright gnomon (note the presence of 'nomo') casts a moving shadow, which thus acts as the isopleth, on the face of the sundial. A set of nomographs for predicting fire behaviour in vegetation[32] demonstrates an application which would likely prove helpful in emergency situations.

A recent phenomenon has been the appearance in medical practice of nomograms (the favoured term in this area) for prognosis, on a statistical basis using Bayes's Theorem, of disease outcomes following a series of clinical tests. Many of these graphical tools do not use true nomographs,[33] but true nomographs for these purposes have recently been developed.[3, 34] 
This is an Accepted Manuscript of an article published by Taylor \& Francis in International Journal of Mathematical Education in Science and Technology on 26/09/2018 available online at:

https://www.tandfonline.com/doi/full/10.1080/0020739X.2018.1527406

\section{Nomography Generation Programs and Sources}

Simple nomographs may be prepared by hand, $[35,36]$ as was necessarily done by the pioneers of nomography, by plotting on coordinate graph paper and with a calculator to hand; however, this can be a somewhat difficult and time-consuming task. PyNomo[10] has been mentioned here as the most sophisticated program available for the preparation of nomographs, but requires some expertise for a successful outcome. Project RHO[37] has elaborate instructions for preparing nomographs (generic, but directed towards use in war gaming!). While many of the early texts on nomography are now out of print, one collection for chemical processing[38] is available, while many hundreds for a variety of purposes can be found by online search.

\section{Summary and Conclusions}

We have here introduced nomographs as convenient and rapid graphical means of performing calculations, even of some complexity. Although nomographs are generally regarded as obsolete, they can be of considerable service in situations requiring reliable results with urgency, as in clinical situations, or for repeated survey over a range. They are not subject to misplacement of decimal points in a calculator, mistaken application of formulae, software obsolescence, poor or absent electronic communication access, temporary loss of power, or absence of power in remote locations. For these and similar reasons, nomographs should be carefully considered as practical tools in appropriate circumstances. 
This is an Accepted Manuscript of an article published by Taylor \& Francis in International Journal of Mathematical Education in Science and Technology on 26/09/2018 available online at:

https://www.tandfonline.com/doi/full/10.1080/0020739X.2018.1527406

\section{Online Resource Files}

Online Resource 1 is an Excel file which demonstrates interactive nomograph use in the calculation of arithmetic means, geometric means, harmonic means, and $\mathrm{Z}$ charts for graphical division. Online Resource 2 is a Python file (in standard text format) which is used with PyNomo to generate Figure 6. Online Resource 3 contains surface and contour plots of a BMI chart, complementing the BMI nomograph of Fig. 6. 


\section{Bibliography}

1. Moler C. "Jahnke and Emde, Revisited" 2014 [August, 2018]. Available from: http://blogs.mathworks.com/cleve/2014/12/15/jahnke-and-emde-revisited/

2. Jahnke E, Emde F. Tables of Functions With Formulas and Curves [Funktionentafeln mit Formeln und Kurven]. 4th ed. New York: Dover; 1945.

3. Marasco J, Doerfler R, Roschier L. Modern Nomograms 2012 [August, 2018]. Available from: http://www.myreckonings.com/modernnomograms/

4. Chung WD, Jr., PROJECT RHO. Nomography Introduction 2008 [August, 2018]. Available from: http://www.projectrho.com/nomogram/intro.html

5. Wikipedia. Nomogram [August, 2018]. Available from: https://en.wikipedia.org/wiki/Nomogram

6. National Institute for Standards and Technology. NIST Digital Library of Mathematical Functions 2016 [August, 2018]. Available from: http://dlmf.nist.gov/

7. OEIS Foundation. The On-Line Encyclopedia of Integer Sequences ${ }^{\mathrm{TM}}$ (OEIS $^{\mathrm{TM}}$ ) [August, 2018]. Available from: http://oeis.org/

8. Evesham HA. Origins and Development of Nomography. Annals of the History of Computing. 1986;8(4):324-333. doi: 10.1109/MAHC.1986.10059.

9. Hankins T, L. Blood, Dirt, and Nomograms: A Particular History of Graphs. Isis. 1999;90(1):50-80.

10. Roschier L. PyNomo - Nomographs with Python [August, 2018]. Available from: http://pynomo.org/wiki/index.php?title=Main_Page

11. Doerfler R. Creating Nomograms with the PyNomo Software 2009 [August, 2018]. Available from: http://www.myreckonings.com/pynomo/CreatingNomogramsWithPynomo.pdf

12. Wikipedia. Centrifuge [August, 2018]. Available from: https://en.wikipedia.org/wiki/Centrifuge

13. Rasmussen HN. Nomogram for Calculation of Centrifugal Data. Eur J Biochem. 1973;34(3):502-505. doi: 10.1111/j.1432-1033.1973.tb02786.x.

14. Ultramagic Balloons. Load Chart [August, 2018]. Available from: http://ultramagic.com/ openfiles/Manuals04/LoadChart. pdf

15. Griffin S. Hot Air Balloon Lift Calculator [(not currently operational)]. Available from: http://www.brisbanehotairballooning.com.au/hot-air-balloon-lift-calculator/

16. Quora. Why won't Java Applets run in my web browser? - see Richard Morris response [August, 2018]. Available from: https://www.quora.com/Why-wont-JavaApplets-run-in-my-web-browser

17. Sigma-Aldrich. Pressure-Temperature Nomograph [August, 2018]. Available from: http://www.sigmaaldrich.com/chemistry/solvents/learning-center/nomograph.html

18. Beale ESL. Conversion of boiling-points of petroleum fractions. In: Dunstan AE, Nash AW, Brooks BT, et al., editors. The science of petroleum. A Comprehensive treatise of the principles and practice of the production, refining, transport and distribution of Mineral Oil. Vol. II. London, New York, Toronto: Oxford University Press; 1938. p. 1280-1286.

19. Nelson KL. Correcting observed boiling points to standard pressure. Am Lab. 1984;16(8):14-27. PubMed PMID: WOS:A1984TD32900002.

20. Doerfler R. The Art of Nomography I, II, and III 2008 [August, 2018]. Available from: http://myreckonings.com/wordpress/2008/01/09/the-art-of-nomography-igeometric-design/ 
This is an Accepted Manuscript of an article published by Taylor \& Francis in International Journal of Mathematical Education in Science and Technology on 26/09/2018 available online at:

https://www.tandfonline.com/doi/full/10.1080/0020739X.2018.1527406

21. Doerfler R. The Lost Art of Nomography: UMAP Journal; 2009 [August, 2018]. Available from: http://www.myreckonings.com/wordpress/2010/04/18/nomographyarticle-in-the-umap-journal/

22. Tournès D. Mathematics of nomography [August, 2018]. Available from: https://www.academia.edu/30868186/Mathematics_of_Nomography

23. Menzel DH. Nomograms. Fundamental Formulas of Physics, Volume 1. 2nd corrected ed: Dover Publications; 2011.

24. University of Texas Health Science Center. Nomogram for body mass index San Antonio, TX[August, 2018]. Available from: http://teachhealthk12.uthscsa.edu/sites/teachhealthk-

12/files/activity/downloads/Nomogram\%20for\%20Body\%20Mass\%20Index \%20Stud ent\%20Handout\%20\%204B.pdf

25. Callaghan LC, Walker JD. An aid to drug dosing safety in obese children: development of a new nomogram and comparison with existing methods for estimation of ideal body weight and lean body mass. Anaesthesia. 2015;70(2):176182. doi: 10.1111/anae.12860.

26. Thimbleby H, Williams D, editors. Using Nomograms to Reduce Harm from Clinical Calculations. 2013 IEEE International Conference on Healthcare Informatics; 2013 911 Sept. 2013.

27. Chemistry World. University fined in caffeine overdose case: Royal Society of Chemistry; [August, 2018]. Available from: https://www.chemistryworld.com/news/university-fined-in-caffeine-overdosecase/2500343.article

28. Wikipedia. Philip Hagar Smith [August, 2018]. Available from: https://en.wikipedia.org/wiki/Phillip_Hagar_Smith

29. Ferry D. Smith Chart 2010 [August, 2018]. Available from: http://www.excelhero.com/blog/2010/08/excel-high-precision-engineering-chart$\underline{1 . h t m l}$

30. Antenna-Theory.com. The Smith Chart [August, 2018]. Available from: http://www.antenna-theory.com/tutorial/smith/chart.php

31. Hart G. Sundials: University of Cambridge; 1999 [August, 2018]. Available from: http://www.sites.hps.cam.ac.uk/starry/ptolsund.html

32. Dimitrakopoulos AP, Dritsa S. Novel nomographs for fire behaviour prediction in Mediterranean and submediterranean vegetation types. Forestry. 2003 January 1, 2003;76(5):479-490. doi: 10.1093/forestry/76.5.479.

33. Kattan MW, Marasco J. What Is a Real Nomogram? Seminars in Oncology. 2010;37(1):23-26. doi: 10.1053/j.seminoncol.2009.12.003.

34. Marasco J, Doerfler R, Roschier L. Doc, What Are My Chances? The UMAP Journal 2011;32(4):279-298.

35. Winchell $\mathrm{H}$. Alignment chart for calculation of specific gravity Am Miner. 1948;33:353-359.

36. Rybner J. Nomograms. General Electric Review. 1930;3(3):164-179.

37. PROJECT RHO. Computerized Nomogram Plotting 2008 [August, 2018]. Available from: http://www.projectrho.com/nomogram/cad.html

38. Kulwiec RA. Chemical Processing Nomographs. 2nd ed. New York: Chemical Pub. Co.; 1969. (Davis DS, editor.). 